\title{
The effects of signaled reward on sign tracking and response rate
}

\author{
JEAN E. ROBERTS, ROGER M. TARPY, and NANCY COONEY \\ Bucknell University, Lewisburg, Pennsylvania
}

\begin{abstract}
If, while responding on a variable interval schedule, rats are given a brief cue prior to reward, their response rate is markedly lower than the rate for yoked partners who receive the cue randomly with respect to reward. This signaled-reward phenomenon has been explained in terms of sign tracking. Two experiments reported here replicated the phenomenon and examined sign tracking directly through visual inspection of the animals' behavior. Although sign tracking did, indeed, occur more in the signaled reward condition, it did not fully account for the difference in response rates.
\end{abstract}

Rats leverpress for food more slowly if every food reinforcer delivered on a variable interval (VI) schedule is preceded by a brief flash of light (correlated condition) than if the light flashes are given independently of their food and responding (random condition). This decrement in response rate, first demonstrated by Pearce and Hall (1978) and St. Claire-Smith (1979), has been cited as an example of stimulus-response overshadowing. That is, for the correlated subjects, the S-US association is said to overshadow the R-US association because the stimulus is a more reliable predictor of food: the stimulus is always followed by food, whereas the response is only infrequently followed by the reinforcer. Although the overshadowing interpretation of this phenomenon is a plausible and appealing one, primarily because it applies the Pavlovian concept of overshadowing to the area of instrumental learning, certain problems have been noted and alternative explanations have been proposed (e.g., Roberts, Tarpy, \& Lea, 1984; Tarpy, Lea, \& Midgley, 1983; Tarpy, Roberts, Lea, \& Midgley, 1984; Williams \& Heyneman, 1982).

One alternative explanation for this signaled-reward effect is based on the concept of sign tracking (see Karpicke, Christoph, Petersen, \& Hearst, 1977; Wasserman, Franklin, \& Hearst, 1974). According to this argument, animals in the correlated condition orient towards and physically contact the light because of its association with food reward. If sign tracking is incompatible with leverpressing, then the reduction in response rate for the correlated group could be due to the fact that the subjects' sign tracking draws them away from the lever and thus competes with leverpressing.

The sign-tracking explanation of the signaled-reward phenomenon was not supported in a number of studies. First, the difference in rate between correlated and random subjects has been observed even when the correlated

Reprints may be obtained from either J. E. Roberts or R. M. Tarpy at the Department of Psychology, Bucknell University, Lewisburg, PA 17837. cue was a noise (Pearce \& Hall, 1978, Experiment 3; St. Claire-Smith, 1979; Williams, 1978). Since noise is not as easily located as a light, the correlated subjects could not have oriented toward the food cue. Second, Hall (1982, Experiment 2) used a procedure in which two response keys were present, and reinforcement was occasionally delivered for responding on either key. A light was correlated with those rewards that were produced by responding on one key but not those produced by responding on the other. Hall reasoned that sign tracking should compete generally with both responses, but he reported that responding was reduced only on the correlated key. Third, in Tarpy et al.'s (1983) first experiment, two groups of rats were trained under a continuous reinforcement (CRF) schedule with either correlated or random cue presentation. A sign-tracking account of the signaledreward phenomenon would predict the usual rate decrement in the correlated condition, whereas an overshadowing account would predict no group differences, because the correlated stimulus and the response predict food equally well. No rate difference was observed.

Sign tracking is still a viable hypothesis despite the work cited above. In the case of the Tarpy et al (1983) experiment, there is a possibility that group differences were not obtained because of either a floor effect or, paradoxically, a ceiling effect. In the case of the former, rates are extremely low on a CRF schedule because subjects spend so much time retrieving and eating the food pellets. Regarding the latter, maximum learning levels are so easily obtained on a CRF schedule that group differences could be masked. Tarpy et al. posed a statistical argument against both of these effects, but they could not offer any more direct evidence against them. In the case of Hall's finding described above, it is possible that a conditional discrimination was acquired such that the cue elicited sign tracking only during responding on the key associated with the correlated cue. Finally, in the case of the experiments utilizing noise as the cue, Holland (1980) has reported that competing locomotor reactions, specifically reactions toward the food source, can be 
elicited by diffuse CSs. Behaviors directed toward the CS can also be sustained, although apparently not generated, by tone CSs (Schwartz, 1973). Thus, sign tracking might still account for the signaled-reward effect.

A number of reported findings are consistent with the sign-tracking hypothesis. First, the cue is known to serve as a secondary reinforcer for subjects in the correlated condition (Pearce \& Hall, 1978, Experiment 4; St. ClaireSmith, 1979); there is no procedural or theoretical reason to suspect that it is not also a viable Pavlovian CS that would elicit sign tracking. Second, Tarpy et al. (1984) observed that less responding occurred in the presence of the cue for the correlated group (although this difference could not account for the overall C vs. R difference), suggesting that sign tracking could have been competing with leverpressing, at least when the light was on. Third, rats have been shown to explore areas of their testing environment that had previously contained a reward-related cue (Albert \& Mah, 1972). This finding indicates that sign tracking is not necessarily dependent upon the continued presence of the cue. Similarly, Iversen (1981) presented a light cue during a 2 -sec delay of reward and observed significant orientation toward the light both during the cue and at other times as well.

The only way to resolve this issue would be to look directly at the sign-tracking behavior within the context of the signaled-reward phenomenon. In the present investigation, we trained rats to press a lever under a VI schedule with either correlated or random cue presentations, that is, conditions typical in demonstrations of the signaled-reward effect. The sessions were recorded on videotape and later scored for sign-tracking behavior. If the sign-tracking account of the signaled-reward phenomenon is correct, then more sign tracking should be observed in the correlated condition. In addition, if competition from sign tracking is the sole reason for the decreased response rate in the $\mathrm{C}$ condition, the response rate during periods not including any sign tracking should be the same in the $\mathrm{C}$ and $\mathrm{R}$ conditions.

\section{EXPERIMENT 1}

\section{Method}

Subjects. Sixteen Long-Evans rats bred in our colonies served as subjects. They weighed between 226 and $278 \mathrm{~g}$ at the start of the experiment and were given free access to water throughout. The rats were housed individually in standard wire-mesh cages in a room with a 12 -h-on/12-h-off light/dark cycle, with experimental sessions taking place during the light cycle. The animals were deprived of food $24 \mathrm{~h}$ prior to the first session and thereafter, following each session, were fed a quantity of food sufficient to maintain their bodies at about $85 \%$ of their free-feeding weights.

Apparatus. Eight identical lever boxes were used. Each was manufactured by Coulbourn Instruments and measured $30 \mathrm{~cm}$ long $\times 25.5 \mathrm{~cm}$ wide $\times 29 \mathrm{~cm}$ high. The boxes were housed in separate sound-attenuating chambers equipped with a ventilating fan. The lever in each box was located directly over the Coulbourn food trough in the center of the front wall ( $12 \mathrm{~cm}$ above the grid floor). Three jewel lights were positioned $3.5 \mathrm{~cm}$ above the lever; the middle (amber) light was used as the cue in this study. In addition, a houselight was centered at the top of the front wall $2 \mathrm{~cm}$ below the ceiling. Rewards were $45-\mathrm{mg}$ food pellets (P. J. Noyes Co., standard diet). The experiment was programmed by an Apple computer located in an adjacent room. Two Sony Betamax video recorders were employed to videotape the rats. When the tapes were played back, observers recorded events using the Apple computer.

Procedure. The rats were pretrained to leverpress in three sessions in which food was delivered according to a variable-time 20-min schedule (VT 20). In addition, leverpresses were reinforced according to a fixed-interval (FI) 5 -sec schedule. Sessions terminated after 100 pellets had been earned or after $5 \mathrm{~h}$. During subsequent sessions, the doors of the outer chambers were left open. This was done in order for conditions to be as similar as possible to those in effect during videotaping, when it was necessary to open the door. Two 3-h sessions, employing a variable-interval (VI) 15-sec schedule of reinforcement, were carried out in this manner before the experimental conditions were instituted. Two animals failed to ac quire the leverpress response and were eliminated from the study. Thus, 14 animals remained as subjects.

Eight daily 20 -min sessions followed the pretraining described above. Two major groups (correlated and random) were designated at this point in accordance with the procedure used by Tarpy et al. (1983). In particular, the correlated (C) subjects received a .5-sec light cue just prior to each reinforcer, that is, between the effective response and the pellet delivery. Whenever a given correlated subject received the light flash, the cue was also delivered simultaneously to its yoked partner. Thus, the random (R) subjects received the same number of lights in the same temporal pattern as the $\mathrm{C}$ subjects, but the lights were independent of their behavior and of reward. $\mathrm{R}$ subjects were not given a light flash when they were reinforced but an equivalent .5-sec delay interval was imposed in order to insure that the temporal relationship between the response and food was equivalent for both groups. The apparatus was physically arranged such that the correlated and yoked boxes were not adjacent to one another. In the first session, the reinforcement schedule in effect was VI $.5 \mathrm{~min}$ (range $=5-55 \mathrm{sec}$ ). On the remaining 7 days of training, a VI 1-min schedule (range $=5-115 \mathrm{sec}$ ) was used.

During each of the eight experimental sessions, two yoked pairs of animals were videotaped. Each animal was videotaped twice, once during the first 4 days of training and again during the final 4 days, 3 to 5 days after the first taping.

Observers. All videotapes were observed and scored separately by each of the three authors. At the time the tapes were scored, one of the observers was completely naive to the experimental conditions and the purpose of the study.

Scoring of videotapes. Two types of behavior were examined when the videotapes were observed. Goal tracking was defined as an insertion of the nose into the food trough. Sign tracking was defined as an orientation toward the light, with the nose being within approximately $2 \mathrm{~cm}$ of it. Note that either of these behaviors could occur at any time, regardless of the presence or absence of stimuli such as food or the light. Observers recorded the behaviors whenever they occurred by pressing the appropriate keys on a microcomputer that was programmed to record the frequency and duration of each class of behavior.

\section{Results}

Figure 1 shows the mean response rates for animals in the $C$ and $R$ conditions across sessions. Although the typical signaled-reward effect was evident, the difference in response rates between the two groups did not reach statistical significance $[F(1,12)=2.1, \mathrm{p}>.05]$, nor did the $C$ versus $R$ treatment interact with sessions $(F<1.0)$. The only significant effect was that due to sessions $[F(7,84)=9.9, p<.01]$. However, if we compare $C$ versus $R$ by analyzing only the data obtained during the 


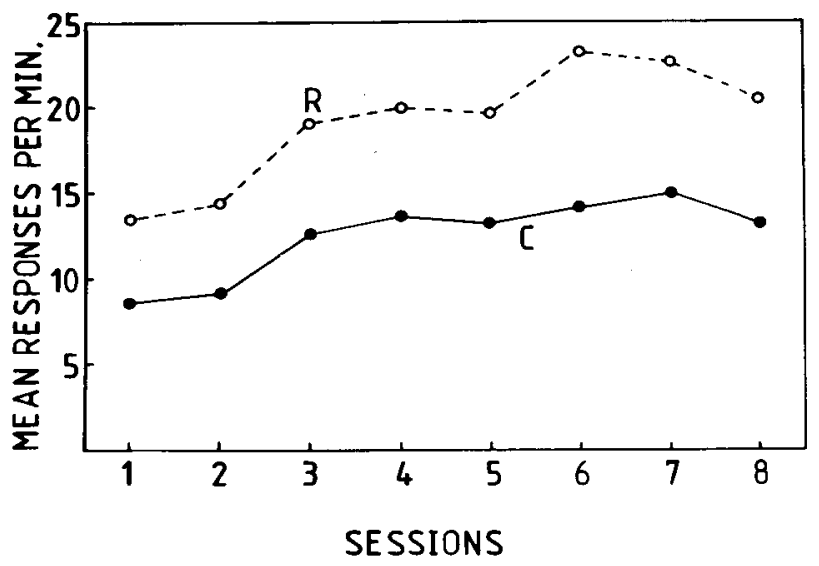

Figure 1. Mean responses per minute for the $C$ and $R$ groups as a function of the eight acquisition sessions in Experiment 1.

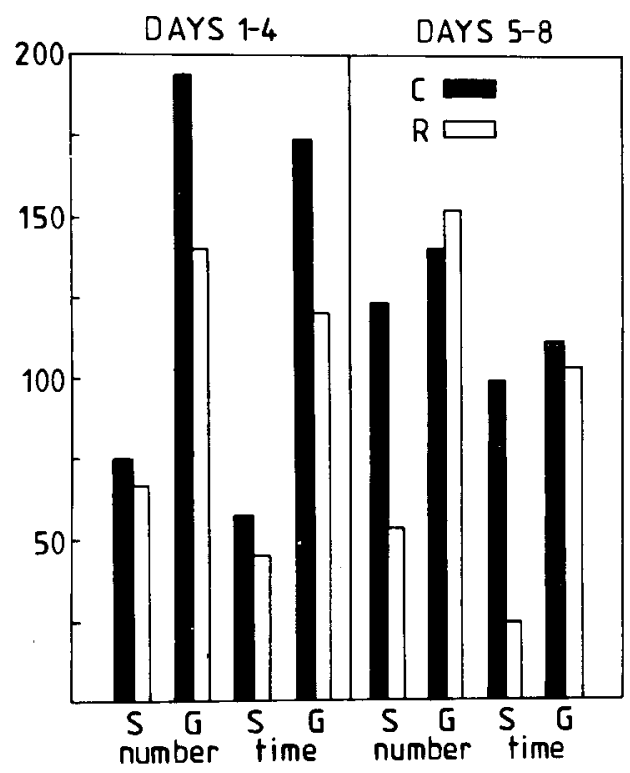

Figure 2. Mean number of responses and mean cumulative time (seconds) for sign (S) and goal (G) responses for the $C$ and $R$ groups during the first (Days 1-4) and last (Days 5-8) videorecorded sessions in Experiment 1. Each subject's number and time represents the mean of three observers' scores. Values on the ordinate refer to both number of responses and cumulative time.

videotaping sessions (two yoked pairs of animals each day), the response rates of Groups $C$ and $R$ did not differ significantly during the first four sessions, but were significantly different during the last four sessions $[\mathrm{t}(12)$ $=2.0, \mathrm{p}<.05$, one-tailed test $]$.

There was good agreement in the data obtained by the three observers in scoring the videotapes. Pearson's $r$ correlation coefficients were computed between all possible pairs of observers for each type of data (number of sign- and goal-tracking responses, cumulative sign- and goal-tracking time). Values ranged from .86 to .98 (median $=.92$ ). Thus, all analyses below employed the mean values of the three observers' data.
The sign and goal tracking data are shown in Figure 2. During the first four sessions, the sign-tracking behavior of the two groups of animals did not differ, either in terms of the number of responses or in terms of the total time spent near the light. It appears from Figure 2 that the $\mathrm{C}$ animals did more goal tracking, but neither the difference in number of responses nor the difference in time spent with the nose in the pellet tray was statistically significant. This pattern changed dramatically during the last four sessions, however. There was still no difference between the two groups in their goal tracking behavior, but the animals in the $\mathrm{C}$ condition made significantly more sign-tracking responses $[t(12)=2.8, p<.02$, two-tailed test] and they spent a greater total time sign tracking [ $\mathrm{t}(12)$ $=3.2, \mathrm{p}<.01]$.

In order to determine whether the difference in response rates between the two groups could be accounted for solely in terms of sign tracking, a corrected response rate was computed for each animal on the 2nd day they were videotaped (Sessions 5-8). A corrected rate was defined as the number of responses during the session divided by the total amount of time spent engaged in behavior other than sign tracking (i.e., session time minus cumulative signtracking time). The means of the corrected rates were 14.1 and 23.4 responses/min for the $C$ and $R$ subjects, respectively. This difference was still statistically significant $[\mathrm{t}(12)=1.8, \mathrm{p}<.05$, one-tailed test $]$. Thus, although the $\mathrm{C}$ animals engaged in significantly more sign-tracking behavior than the $\mathrm{R}$ animals, such behavior alone cannot account for the $C$ versus $R$ rate difference, since the difference was obtained for corrected scores as well as for the uncorrected values.

In summary, at the same time that the typical response rate difference emerged (Days 5-8), the correlated animals were also spending more time inspecting the food cue. The sign-tracking hypothesis would predict this outcome. A difference in response rate between the two groups still remained, however, at times when sign tracking was not occurring.

\section{EXPERIMENT 2}

The results of Experiment 1 might be called into question because the typical difference in response rate between the $C$ and $R$ groups was not statistically significant when all the data were considered. If we are to dismiss the sign-tracking hypothesis as the cause of the signaledreward effect, then it would seem important that we show a stronger and more reliable $\mathrm{C}-\mathrm{R}$ rate difference. We believe that the difference was not reliable because of the disruptive effects of having the doors of the operant chambers open during the session, especially on the early critical training days. Experiment 2, therefore, attempted to overcome this problem by beginning training with the doors closed and then gradually opening them once the typical rate difference was established. All animals were then videotaped for a short period of time on the same day in attempt to replicate the basic findings of Experiment 1 . 


\section{Method}

Subjects and Apparatus. Sixteen male Long-Evans rats bred in our colonies served as subjects. They weighed between 384 and $450 \mathrm{~g}$ at the start of the experiment, and were maintained as in Experiment 1 . One animal failed to press the lever during pretraining and was therefore discarded from the experiment; this left 15 subjects. The apparatus was the same as that used in Experiment 1.

Procedure. Following the pretraining phase, 12 daily 30 -min sessions were carried out, with seven animals randomly assigned to the correlated condition and eight to the random condition. The contingencies and yoking procedures were the same as in Experiment 1 , with reinforcement delivered on a VI .25-min schedule on Day 1 and a VI 1-min schedule thereafter. The doors of the chambers remained fully closed until the 6th session, when they were opened half way. Beginning on the 8th day, the doors were fully open during the session.

On the 13th (test) day, all animals were run, one at a time, for $10 \mathrm{~min}$ (the reason for this minor change in procedure was that we had only one video camera available to us at the time of this experiment). Within each yoked pair, the $\mathrm{C}$ animal was run first so that the experimenters could subsequently deliver to the $\mathrm{R}$ subject an equal number of light flashes as had been earned by the $C$ animal . Each animal's session was videotaped, as in Experiment 1. The second $5 \mathrm{~min}$ of each videotape was later scored by each of the three observers as in Experiment 1, one observer still being naive to the experimental conditions and purpose of the study.

\section{Results}

Figure 3 shows the mean response rates for the $\mathrm{C}$ and $\mathrm{R}$ subjects during the 12 sessions when the VI 1-min schedule of reinforcement was in effect; the last point represents the data from the videotaped test session. Analyses of variance were carried out using data from the last four sessions (after the doors were opened) and using all 12 days' data. In both cases, the difference between Groups $C$ and $R$ was statistically significant $[F(1,13)$ $=7.5, \mathrm{p}<.05 ; \mathrm{F}(1,13)=6.0, \mathrm{p}<.05$, for 4 and 12 sessions, respectively]. The effects due to sessions $[\mathrm{F}(3,39)=8.5, \mathrm{p}<.01 ; \mathrm{F}(11,143)=5.2, \mathrm{p}<.01]$ and to the interaction of groups and sessions $[\mathrm{F}(3,39)=$ $5.4, p<.01 ; F(11,143)=2.4, p<.05]$ were also statistically significant in both cases. Finally, the rate differ-

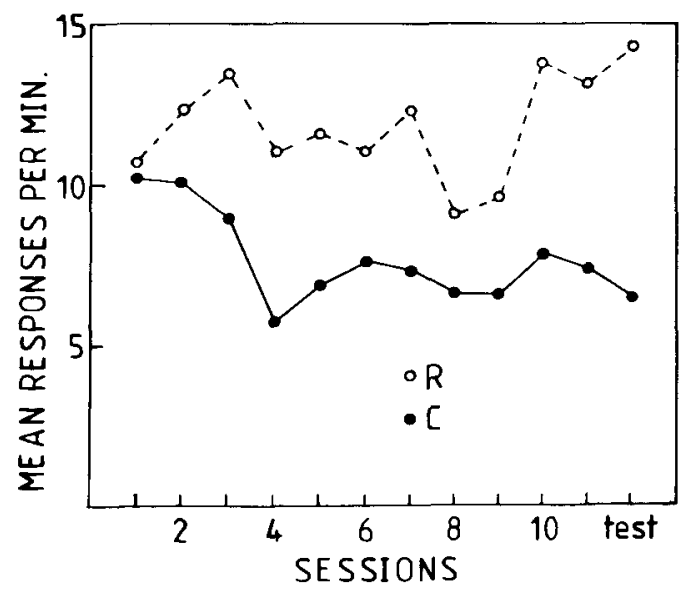

Figure 3. Mean responses per minute for the $C$ and $R$ groups on the 12 acquisition sessions in Experiment 2. Day 12 was the test (videorecorded) session.

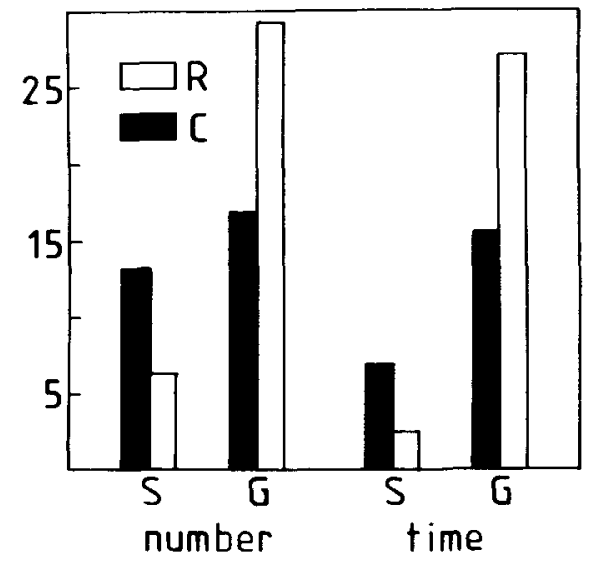

Figure 4. Median number of responses and median cumulative time (seconds) for sign (S) and goal (G) responses for the $\mathbf{C}$ and $\mathrm{R}$ groups during the test session in Experiment 2. Each subject's number and time score represents the mean of three observers' scores. Values on the ordinate refer to both number of responses and cumulative time.

ence on the test day was also statistically significant [t(13) $=3.1, \mathrm{p}<.01$, two-tailed test $]$.

In analyzing the videotape-scoring data from the test day, we followed the same procedure used in Experiment 1 by using the mean of the three observers' scores to represent an animal's sign- and goal-tracking behavior. One animal in Group $\mathbf{R}$ had an extremely high signtracking score, which caused group variability to be markedly unequal (the animal's score was nearly 20 standard deviations from the mean of its group). Thus, nonparametric (Mann-Whitney U) statistical tests were used for the analysis of sign- and goal-tracking behavior across groups.

Figure 4 shows the median scores of the $C$ and $R$ animals for both sign tracking (number of responses and cumulative time in seconds) and goal tracking (number of responses and cumulative time). The results replicate those from Experiment 1: significantly more signtracking behavior occurred for animals in the correlated than in the random group, both in terms of the number of responses $(U=13, p=.04)$ and in terms of the total amount of time spent inspecting the cue $(U=11$, $p=.02$ ). On the other hand, there was no significant difference between the groups for goal tracking ( $U=18$ and $\mathbf{1 7 . 5}$ for number of responses and cumulative time, respectively), despite the higher median values for Group $R$ indicated in Figure 4.

Individual response rates on the test day were each corrected for time spent sign tracking, as in Experiment 1. (We assumed that an equal amount of time was spent sign tracking in each half of the 10-min session. Thus, total time spent sign tracking was estimated by doubling the time observed in the last $5 \mathrm{~min}$.) In addition, we computed response rates similarly corrected for time engaged in either sign or goal tracking [number of responses divided by session time minus (cumulative sign-tracking time plus cumulative goal-tracking time)]. The rates for 
the $C$ animals were $6.4,6.6$, and 7.1 responses/min when uncorrected, corrected for sign tracking, and corrected for both sign and goal tracking, respectively. The corresponding rates for the $\mathrm{R}$ animals were $14.2,15.0$, and 17.6 responses $/ \mathrm{min}$. The difference between the $\mathrm{C}$ and $R$ animals using the corrected rates was still statistically significant [correcting for sign tracking only: $t(13)=$ $2.66, \mathrm{p}<.02$; correcting for both sign and goal tracking: $\mathfrak{t}(13)=2.81, \mathrm{p}<.02$, two-tailed tests].

\section{GENERAL DISCUSSION}

The two experiments reported here showed directly that sign tracking, or inspection of the site of the food-correlated cue, does indeed occur more in the correlated condition than in the random condition. Furthermore, Experiment 1 showed that Group C sign tracking increased over sessions (i.e., from the first to the last 4 days) at the same time that their rate of leverpressing became lower than that of Group R. These results, therefore, lend credibilty to the sign-tracking explanation of the signaled reward phenomenon. However, a large rate difference between the two groups still remained when the time spent sign tracking was discounted. Thus, competition between leverpressing and sign tracking does not appear to be a sufficient explanation of the phenomenon. The direct observations described here, then, corroborate the indirect evidence against the sign-tracking hypothesis discussed in the introduction.

It should be noted that there is no a priori reason to assume that sign tracking should compete with leverpressing. For example, one might predict that sign tracking would facilitate lever responding if the CS were to draw the subjects nearer to the lever. If this were true, however, then the effect of sign tracking would oppose the usual C-R difference and thus not explain this phenomenon. The fact that the $\mathrm{C}$ animals' corrected rates were somewhat higher than their overall rates suggests that sign tracking did, in fact, compete with leverpressing, but only to a small degree.

Our results are generally in agreement with those of Iversen (1981), who examined sign and goal tracking in animals receiving a 2 -sec signaled delay of reinforcement. $\mathrm{He}$, too, reported a moderate increase in sign tracking at times other than during the delay, although most of the sign tracking in his study occurred in the presence of the cue. Our studies, however, differ from Iversen's in terms of the effect of the signal on goal-directed behavior. Iversen reported an increase in goal tracking with signaled reward, whereas we did not find such an effect using our procedure. It should be noted that most of the goal tracking in Iversen's experiment occurred during the delay; only one of three animals showed a marked increase at other times. Thus, differences between these studies are most likely a result of differences in procedure, namely the length of the delay of reinforcement used.

In summary, sign tracking is indeed more prevalent with signaled reward-as well it should be, given the etiology of sign tracking. However, the present studies provide the first direct evidence indicating that such behavior does not wholly account for the decreased response rate found with signaled reward.

\section{REFERENCES}

Albert, D. J., \& MAH, C. J. (1972). An examination of conditioned reinforcement using a one-trial learning procedure. Learning and Motivation, 3, 369-388.

HALL, G. (1982). Effects of a brief stimulus accompanying reinforcement on instrumental responding in pigeons. Learning and Motivation, 13, 26-43.

Holland, P. C. (1980). Second-order conditioning with and without unconditioned stimulus presentation. Journal of Experimental Psychology: Animal Behavior Processes, 3, 238-250.

IVERSEN, I. H. (1981). Response interactions with signaled delay of reinforcement. Behaviour Analysis Letters, 1, 3-9.

Karpicke, J., Christoph, G., Peterson, G., \& Hearst, E. (1977). Signal location and positive versus negative conditioned suppression in the rat. Journal of Experimental Psychology: Animal Behavior Processes, 3, 105-118.

Pearce, J. M. , \& Hall, G. (1978). Overshadowing the instrumental conditioning of a lever-press response by a more valid predictor of the reinforcer. Journal of Experimental Psychology: Animal Behavior Processes, 4, 356-367.

Roberts, J. E., TARPY, R. M., \& LEA, S. E. G. (1984). Stimulusresponse overshadowing: Effects of signaled reward on instrumental responding as measured by response rate and resistance to change. Journal of Experimental Psychology: Animal Behavior Processes, 10, 244-255.

Schwartz, B. (1973). Maintenance of key pecking by responseindependent food presentations: The role of the modality of the signal for food. Journal of the Experimental Analysis of Behavior, 20, 17-22.

St. Claire-Smith, R. (1979). The overshadowing of instrumental conditioning by a stimulus that predicts reinforcement better than the response. Animal Learning \& Behavior, 7, 224-228.

TARPY, R. M., LEA, S. E. G., \& Midgley, M. (1983). The role of response-US correlation in stimulus-response overshadowing. Quarterly Journal of Experimental Psychology, 35B, 53-65.

TArPy, R. M., Roberts, J. E., Lea, S. E. G., \& Midgley, M. (1984). The stimulus-response overshadowing phenomenon with VI versus FI schedules of reinforcement. Animal Learning \& Behavior, 12 , 50-54.

Wasserman, E. A., Franklin, S. R., \& Hearst, E. (1974). Pavlovian appetitive contingencies and approach versus withdrawal to conditioned stimuli in pigeons. Journal of Comparative and Physiological Psychology, 86, 616-627.

Williams, B. A. (1978). Information effects on the response-reinforcer association. Animal Learning \& Behavior, 6, 371-379.

Williams, B. A., \& HEYNEMAN, N. (1982). Multiple determinants of "blocking"' effects on operant behavior. Animal Learning \& Behavior, 10, $72-76$.

(Manuscript received June 6, 1984; revision accepted for publication October 8, 1984.) 